\title{
Bienestar Psicológico ¿Mejor soltero, en una relación o casado? ${ }^{3}$
}

Diana Camila Garzón Velandia

Magister en Psicología Universidad Católica de Colombia, Colombia Correo electrónico: dcgarzon@ucatolica.edu.co
Recibido: 29/10/2018

Evaluado: 28/12/2018

Aceptado: 25/06/2019

\section{Resumen}

Esta investigación de tipo descriptivo comparativo tenía el objetivo de comparar el Bienestar psicológico -BP- entre un grupo de 92 personas solteras, 71 personas que se encontraban en una relación y 52 personas casadas 0 en unión libre, con edades entre 18 a 55 años. Se aplicó la escala multidimensional de bienestar psicológico de Ryff (1989a) que evalúa seis dimensiones de bienestar psicológico (dominio del entorno, propósito de vida, autonomía, autoaceptación, crecimiento personal y relaciones positivas con otros). Se compararon los puntajes medios de muestras independientes, ante lo cual no se evidenciaron diferencias estadísticamente significativas en el bienestar psicológico entre personas solteras, en una relación y casadas, considerando un nivel de confianza de 95\%, sin embargo, cuando se reduce el nivel de confianza de $93 \%$, se determinan diferencias entre las personas solteras y las personas que se mantienen en una relación, posicionando las primeras sobre las demás; de igual manera, la interpretación de los resultados permitió identificar diferencias en las dimensiones de bienestar psicológico que posiciona a las personas de los diferentes grupos por encima de las otras, según la dimensión.

\section{Palabras clave}

Bienestar psicológico, Estado sentimental, psicología positiva.

3 Para citar este artículo: Garzón-Velandia, D.C. (2020). Bienestar Psicológico ¿Mejor soltero, en una relación o casado?. Informes Psicológicos, 20(2), pp. 41-53 http://dx.doi.org/10.18566/infpsic.v20n2a3 


\section{Psychological well-being: Is it better to be single, in a relationship or married?}

Abstract

This comparative descriptive research had the objective of comparing the psychological well-being (PWB) among a group of 92 single people, 71 people who were in a relationship and 52 people who were married or in cohabitation, with ages ranging between 18 to 55. Ryff's (1989a) multidimensional scale of psychological well-being was applied, which evaluates six dimensions of psychological well-being (environmental mastery, purpose in life, autonomy, selfacceptance, personal growth, and positive relationships with others). The mean scores of independent samples were compared, before which there were no statistically significant differences in psychological well-being between single people, people who were in a relationship or married people, considering a confidence level of $95 \%$. However, when the level of confidence of $97 \%$ is reduced, differences are determined between single people and people who are in a relationship, placing the former over others. Likewise, the interpretation of the results allowed to identify differences in the dimensions of psychological well-being that positions the people of the different groups above the others, according to the dimension.

Keywords Psychological well-being, relationship status, positive psychology.

\section{Bem-estar psicológico. É melhor ficar solteiro, estar em um relacionamento ou estar casado?}

Resumo

Esta pesquisa descritiva comparativa teve como objetivo comparar o bem-estar psicológico - BP - entre um grupo de 92 pessoas solteiras, 71 pessoas que estavam em um relacionamento e 52 pessoas casadas ou em união livre, com idades entre 18 e 55 anos. Foi aplicada a escala multidimensional de bem-estar psicológico de Ryff (1989a), que avalia seis dimensões do bem-estar psicológico (domínio do entorno, objetivo da vida, autonomia, auto-aceitação, crescimento pessoal e relações positivas com os outros). Foram comparados os escores médios das amostras independentes, em face do qual não evidenciaram-se diferenças estatisticamente significativas no bem-estar psicológico entre as pessoas solteiras, em um relacionamento ou casadas, levando em consideração um nível de confiança de 95\%, porém, quando se reduz o nível de confiança do $97 \%$, determinaram-se diferenças entre as pessoas solteiras e as pessoas que permanecem em uma relação, posicionando as primeiras por cima das outras. Do mesmo jeito, a interpretação dos resultados permitiu identificar diferenças nas dimensões do bem-estar psicológico que posiciona às pessoas de um grupo por cimas das outras, em concordância com a dimensão.

Palavras chave Bem-estar psicológico, estado sentimental, psicologia positiva. 


\section{ntroducción}

Desde el matrimonio a la soltería existe una tipología extensa y variada de relaciones de pareja con diferentes grados de formalización y estabilidad. Las relaciones sentimentales han cambiado en las últimas décadas, sin embargo, el matrimonio sigue siendo una tendencia en la actualidad. Aunque sean aceptados múltiples tipos de convivencia entre las parejas, los matrimonios son el tipo de vínculo que se ha mantenido a lo largo del tiempo (Superintendencia de Notariado y Registro, 2014; 2017).

Los múltiples tipos de relaciones de pareja han sido estudiadas desde diferentes enfoques y campos de la psicología, como la psicología social (García \& Nader, 2009), la psicología clínica (Szmulewicz, 2015) o la psicología positiva, la cual ha enfatizado sus investigaciones en las fortalezas y potencialidades de los tipos de pareja desde diferentes modelos y variables (felicidad, bienestar subjetivo, bienestar psicológico, salud), siendo el matrimonio y la cohabitación o la unión de hecho los tipo de pareja más estudiados (Erhabor \& Neo, 2013; Jeong-Hwa, 2015; Pozos, López, Rivera, Vargas \& Reidl, 2013; Soulsby \& Bennett, 2015).

En tales investigaciones se ha identificado una clara evidencia de la relación existente entre la salud física y psicológica con una sana relación de pareja. Igualmente, una perspectiva diferencial menciona que las mujeres tienden a ser más felices que los hombres en el matrimonio, pues estos experimentan una tendencia al decremento de la satisfacción vital a lo largo del matrimonio. No obstante, las investigaciones parecen ser concluyentes cuando se relaciona una unión marital tradicional con el bienestar, incluso en diferentes culturas, por ejemplo occidentales, africanas y medio orientales (Azam, 2012; Chapman \& Guven, 2014; Erhabor \& Neo, 2013).

Sin embargo, la evidencia no se dirige en la misma dirección cuando de comparar personas solteras y casadas se trata, puesto que algunas investigaciones concluyen que las personas casadas presentan altos niveles de bienestar en contraste con las personas solteras, incluso, en contraste con personas que cohabitan y personas viudas (Shapiro \& Keyes, 2008; Simó-Noguera, González-Sanjuán, Muñoz-Rodríguez, Hernández-Monleón, 2015; Soulsby \& Bennett, 2015). Empero, otras investigaciones mencionan que el bienestar y felicidad de las personas solteras es igual y, en ocasiones, superior a las personas casadas, con base en significancias menores a .05 y magnitudes mayores a 19 (Girme, Overall, Faingataa \& Sibley, 2015; Jeong-Hwa, 2015; Musick \& Bumpass, 2012).

De acuerdo con ello, en otras investigaciones se menciona que podría no existir una relación significativa entre el matrimonio y la felicidad, puesto que las personas solteras no relacionan la felicidad con un matrimonio futuro, lo cual es consistente con conclusiones que evidencian que las personas prefieren estar solteras y felices, y no vivir un mal matrimonio (Chapman \& Guven, 2014). Sin embargo, es menos probable que las personas permanezcan solas si encuentran la oportunidad de tener una relación que aporte felicidad, lo que implica que la calidad del vínculo -que se ve influida por 
variables como la educación, la edad, los ingresos y la salud- es un factor determinante en el bienestar y la felicidad de una pareja (Girme et al., 2015; Mikucka, 2016; Sheck, 1995; Tze-Li, 2014).

A pesar de que gran parte de la evidencia es contradictoria, uno de los factores en los cuales la mayoría de las investigaciones puntualiza gran relevancia son las relaciones cercanas con otras personas, sea su pareja o demás personas que generan una red de apoyo emocional, poniendo en duda si la importancia del bienestar radica en la situación sentimental o en la calidad de las relaciones sociales. De acuerdo con ello, el modelo multifactorial de bienestar psicológico de Ryff resulta apropiado para el estudio del bienestar psicológico desde una perspectiva comparativa que permita determinar el bienestar entre personas casadas y solteras, dado que, entre las dimensiones que lo componen, se determinan las relaciones positivas con otros (Ryff, 1989a).

Dicho modelo define el bienestar psicológico como el esfuerzo por perfeccionarse y por lograr la realización del propio potencial, el cual se compone de seis factores: control ambiental, como la habilidad para transformar el ambiente en pro de satisfacer sus necesidades y deseos; crecimiento personal, como una dinámica de aprendizaje continuo; propósito de vida, que unifica los esfuerzos y retos de las personas; autonomía, como un sentido de individualidad y libertad personal; autoaceptación, es decir, hallarse agradable estando consciente de sus limitaciones; y relaciones positivas con otros, caracterizadas principalmente por ser relaciones cálidas y cercanas. Tales dimensiones no son variables predictoras, sin embargo, resultan ser un indicador del funcionamiento óptimo o del funcionamiento deficitario de las personas (Ryff, 1989b; 2004; Vázquez, \& Hervás, 2012).

Con base en el modelo Multifactorial de Bienestar Psicológico, la presente investigación tiene como objetivo comparar el bienestar psicológico entre un grupo de personas solteras y un grupo de personas casadas o que cohabiten con su pareja de manera permanente, para responder a la pregunta: ¿Existe diferencia en el bienestar psicológico entre personas casadas, que mantienen una relación de pareja y personas que no mantienen una relación de pareja?, la cual fue formulada con el fin de aportar evidencia que brinde soporte empírico y que facilite reconocer el impacto real de convivir con una pareja o no, sobre el bienestar psicológico y ayude a mitigar la discrepancia entre la evidencia encontrada sobre las variables en mención.

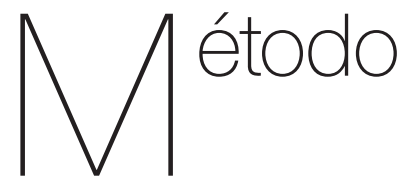

\section{Diseño y participantes}

La investigación es un estudio descriptivo comparativo transversal de corte cuantitativo, en la cual se analizaron los datos de 215 personas seleccionadas por medio de un muestreo no probabilístico por voluntarios, divididos en tres grupos: personas solteras (92 personas que en último año no hubieran tenido una pareja estable como criterio de inclusión), personas en una relación (71 personas que tuvieran una relación mayor a un mes y en la cual no hubiera cohabitación) y 
personas casadas (52 personas casadas y que mantuvieran al menos un año de convivencia con su pareja, como criterio de inclusión), con edades comprendidas entre los 18 y 55 años.

En la Tabla 1 se evidencia que la distribución de la muestra entre sexo y estado sentimental varía entre grupos, teniendo mayor cantidad de mujeres solteras. Otros estadísticos de tendencia central caracterizaron la edad de la muestra, en donde, la media corresponde a 25.7 años, con una desviación estándar de 9.1. cuya edad mínima es de 18 años y la edad máxima de 55 años.

Tabla 1

Frecuencias de estado sentimental y sexo.

\begin{tabular}{ccccc}
\hline & \multicolumn{4}{c}{ Estado sentimental actual } \\
\cline { 2 - 5 } Sexo & Casado & En una relación & Soltero (a) & Total \\
\hline Hombre & 16 & 10 & 30 & 56 \\
Mujer & 36 & 61 & 62 & 159 \\
\hline Total & 52 & 71 & 92 & 215 \\
\hline
\end{tabular}

\section{Instrumentos}

La Escala Multifactorial de Bienestar Psicológico de Ryff (1989b), mide seis factores de bienestar psicológico: autoaceptación, relaciones positivas con otros, autonomía, dominio del entorno, propósito de vida, crecimiento personal y un índice de bienestar global. La escala ha sido validada en población colomboespañola (Díaz et al., 2006) y se compone de 39 ítems en su versión corta, su confiabilidad es de .68 (confiabilidad por dimensiones entre .68 a .83) y su Ajuste al modelo teórico es de .75 .
Adicionalmente, se utilizó una hoja de datos personales evaluada por un juez experto, en donde se preguntó a los participantes sobre la percepción de su relación, la satisfacción personal con respecto a la misma, entre otros datos que describían la relación que mantiene las personas casadas y las personas que se encontraban en una relación.

\section{Procedimiento}

Se contactó a la población por medio de un muestreo por voluntarios teniendo en cuenta variables como sexo, edad y estado sentimental, con el fin de lograr la homogeneidad de la muestra. Luego de explicar los objetivos de la investigación, los participantes firmaron el consentimiento informado y diligenciaron los instrumentos de forma digital, asegurando la anonimización de los datos, de conformidad con las consideraciones éticas dadas por la Ley 1090 de 2006 y el código de ética de la APA. Posterior al análisis de datos, se entregó un informe con los resultados a cada participante que lo solicitó.

\section{Análisis de datos}

Una vez se consolidó la base de datos, se depuró por medio de imputación simple con media para datos perdidos y se obtuvieron estadísticos descriptivos de tendencia central para caracterizar la muestra. Posteriormente, se analizó la normalidad de la variables para identificar la prueba estadística apropiada para la comparación de medias entre grupos; se determinó que las variables 
autonomía y Bienestar psicológico se ajustaba a la distribución normal por medio de la prueba Kolmogorov-Simornov (KS) y un valor $\mathrm{p}=.07$, sin embargo las demás variables no se ajustaron a la distribución normal, por lo cual se realizaron dos análisis: Anova y Kruskal Wallis, con el objetivo de identificar si existían diferencias significativas en el bienestar psicológico en función del estado sentimental actual.

Finalmente, se realizaron análisis complementarios de correlación entre variables como sexo, edad, satisfacción percibida del estado sentimental, tiempo de permanencia en el estado sentimental y tiempo de la relación, para las personas casadas. Los análisis se realizaron con un intervalo de confianza del 95\%.

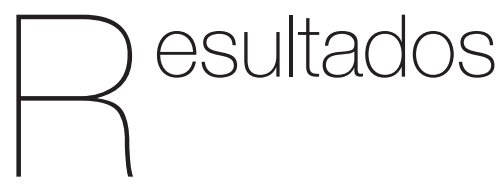

La Tabla 2 muestra las puntuaciones medias de los participantes, agrupadas por su estado sentimental, apreciándose que tanto las personas del grupo Casados como del grupo Solteros tiene, en su mayoría, puntuaciones promedio, lo que muestra un nivel de Bienestar Psicológico aceptable, conforme a los valores estándar de la prueba.

Tabla 2

Estadísticos descriptivos de Bienestar Psicológico agrupado por su Estado Sentimental

\begin{tabular}{|c|c|c|c|c|c|c|c|c|c|c|c|c|c|c|}
\hline & \multicolumn{4}{|c|}{ Casado } & \multicolumn{4}{|c|}{ En una relación } & \multicolumn{4}{|c|}{ Soltero } & \multicolumn{2}{|c|}{ Estándar } \\
\hline & Media & $\mathrm{DE}$ & Min & $\operatorname{Max}$ & Media & $\mathrm{DE}$ & Min & $\operatorname{Max}$ & Media & $\mathrm{DE}$ & Min & $\operatorname{Max}$ & Media & $\mathrm{DE}$ \\
\hline Autoaceptación & 4.39 & .82 & 2 & 6 & 4.53 & .8 & 1.8 & 6 & 4.39 & .8 & 1.5 & 6 & 4.31 & .86 \\
\hline Relaciones positivas & 4.31 & .96 & 1.6 & 6 & 4.42 & .79 & 2.6 & 6 & 4.18 & .9 & 1.8 & 6 & 4.58 & .85 \\
\hline Autonomía & 4.46 & .75 & 2.6 & 6 & 4.41 & .77 & 2.6 & 6 & 4.15 & .8 & 1.7 & 6 & 4.24 & .73 \\
\hline Dominio del entorno & 4.79 & .73 & 3 & 6 & 4.77 & .68 & 2.5 & 6 & 4.55 & .7 & 2.6 & 6 & 4.31 & .72 \\
\hline Crecimiento personal & 4.72 & .64 & 3 & 6 & 5.05 & .64 & 2.5 & 6 & 4.81 & .7 & 3 & 6 & 4.57 & .64 \\
\hline Propósito de vida & 4.61 & .68 & 2.8 & 5.9 & 4.72 & .63 & 2.8 & 5.8 & 4.52 & .5 & 2.9 & 5.7 & 4.47 & .83 \\
\hline Bienestar Psicológico & 4.59 & .64 & 2.7 & 5.8 & 4.68 & .52 & 2.5 & 5.7 & 4.46 & .6 & 2.9 & 5.6 & 4.4 & .77 \\
\hline
\end{tabular}

A partir de la prueba Anova, dada para las variables normales, se determina que no hay suficiente evidencia para asumir diferencia estadísticamente significativa con respecto al Bienestar psicológico entre los grupos, de acuerdo con el valor $p=.064$, y un intervalo de confianza de 95\%, sin embargo, si se reduce el margen de confianza a 93\% se determina que el $p$ valor es significativo. De otra parte, la dimensión de autonomía presenta un p valor menor a .05, por lo cual se aceptan diferencias significativas entre los grupos (ver Tabla 3). 
Tabla 3

Anova de un factor para variables normales en función del estado sentimental

\begin{tabular}{ccccccc}
\hline & & $\begin{array}{c}\text { Suma de } \\
\text { cuadrados }\end{array}$ & gl & $\begin{array}{c}\text { Media } \\
\text { cuadrática }\end{array}$ & $\mathrm{F}$ & Sig. \\
\cline { 2 - 7 } Autonomía & Entre grupos & 4.191 & 2 & 2.096 & 3.464 & $.033^{*}$ \\
\hline Bienestar Psicológico & Entre grupos & 1.925 & 2 & .962 & 2.788 & .064 \\
\hline
\end{tabular}

Nota: * $p<.05$, significancia estadística

Con respecto a las diferencias presentadas en la dimensión autonomía, se desarrolla un análisis post hoc para establecer las diferencias entre grupos, como se evidencia en la Tabla 4. En esta se muestra que la diferencia para la variable autonomía se encuentra en la comparación del grupo de personas casadas y el grupo de personas solteras, en donde, el segundo grupo presenta medias superiores. Por su parte, para la variable Bienestar psicológico, se determina una diferencia significativa entre el grupo de personas que están en una relación y personas solteras, en la cual las personas solteras presentan medias más bajas.

Tabla 4.

Post hoc con prueba Bonferroni para diferencias de medias en grupos de estado sentimental

\begin{tabular}{|c|c|c|c|c|c|c|c|}
\hline & & & \multirow{2}{*}{$\begin{array}{l}\text { Diferencia de } \\
\text { medias (I-J) }\end{array}$} & \multirow{2}{*}{ Desv. Error } & \multirow{2}{*}{ Sig. } & \multicolumn{2}{|c|}{ Intervalo de confianza al $95 \%$} \\
\hline & & & & & & Límite inferior & Límite superior \\
\hline \multirow{6}{*}{ Autonomía } & \multirow{2}{*}{ Casado } & En una relación & .05 & .14 & 1.000 & -.29 & .39 \\
\hline & & Soltero & .31 & .13 & $.070^{*}$ & -.02 & .63 \\
\hline & \multirow{2}{*}{ En una relación } & Casado & -.05 & .14 & 1.000 & -.39 & .29 \\
\hline & & Soltero & .26 & .12 & .109 & -.04 & .56 \\
\hline & \multirow{2}{*}{ Soltero } & Casado & -.31 & .13 & $.070^{\star}$ & -.63 & .02 \\
\hline & & En una relación & -.26 & .12 & .109 & -.56 & .04 \\
\hline \multirow{6}{*}{$\begin{array}{l}\text { Bienestar } \\
\text { Psicológico }\end{array}$} & \multirow{2}{*}{ Casado } & En una relación & -.09 & .11 & 1.000 & -.35 & .17 \\
\hline & & Soltero & .13 & .10 & .644 & -.12 & .37 \\
\hline & \multirow{2}{*}{ En una relación } & Casado & .09 & .11 & 1.000 & -.17 & .35 \\
\hline & & Soltero & .22 & .09 & $.061^{\star}$ & -.01 & .44 \\
\hline & \multirow{2}{*}{ Soltero } & Casado & -.13 & .10 & .644 & -.37 & .12 \\
\hline & & En una relación & -.22 & .09 & $.061^{*}$ & -.44 & .01 \\
\hline
\end{tabular}

Por su parte, para las variables no normales, se realizó una comparación de grupos con la prueba estadística Kruskal Wallis, como se evidencia en la Tabla 5, en la cual se muestran diferencias significativas con un intervalo de confianza de $95 \%$ en las dimensiones de dominio del entorno, cuyas puntuaciones son superiores en el grupo de personas casadas, y crecimiento personal, que tiene mayores puntuaciones en el grupo de personas en una relación. De igual manera, se evidencian diferencias significativas con respecto a la satisfacción de las personas con su situación sentimental, en la cual, las personas casadas mantienen puntuaciones más altas de satisfacción. 
Tabla 5.

Comparación de medias de bienestar psicológico mediante la prueba Kruskal Wallis.

\begin{tabular}{|c|c|c|c|c|}
\hline Estado sentimental actual & & $\mathrm{N}$ & Rango promedio & Sig. asintótica \\
\hline \multirow{4}{*}{ Autoaceptación } & Casado & 52 & 103.81 & \multirow{4}{*}{.478} \\
\hline & En una relación & 71 & 115.30 & \\
\hline & Soltero (a) & 92 & 104.73 & \\
\hline & Total & 215 & & \\
\hline \multirow{4}{*}{ Relaciones positivas } & Casado & 52 & 111.00 & \multirow{4}{*}{.259} \\
\hline & En una relación & 71 & 115.87 & \\
\hline & Soltero (a) & 92 & 100.23 & \\
\hline & Total & 215 & & \\
\hline \multirow{4}{*}{ Dominio del entorno } & Casado & 52 & 117.90 & \multirow{4}{*}{$.041^{*}$} \\
\hline & En una relación & 71 & 116.75 & \\
\hline & Soltero (a) & 92 & 95.65 & \\
\hline & Total & 215 & & \\
\hline \multirow{4}{*}{ Crecimiento personal } & Casado & 52 & 91.58 & \multirow{4}{*}{$.007^{*}$} \\
\hline & En una relación & 71 & 125.68 & \\
\hline & Soltero (a) & 92 & 103.64 & \\
\hline & Total & 215 & & \\
\hline \multirow{4}{*}{ Propósito de vida } & Casado & 52 & 107.94 & \multirow{4}{*}{.098} \\
\hline & En una relación & 71 & 119.97 & \\
\hline & Soltero (a) & 92 & 98.79 & \\
\hline & Total & 215 & & \\
\hline \multirow{4}{*}{$\begin{array}{l}\text { Satisfacción con el } \\
\text { estado sentimental }\end{array}$} & Casado & 50 & 127.43 & \multirow{4}{*}{$.000^{*}$} \\
\hline & En una relación & 71 & 118.10 & \\
\hline & Soltero (a) & 91 & 85.94 & \\
\hline & Total & 212 & & \\
\hline
\end{tabular}

Nota: * $p<.05$, con intervalo de confianza de $95 \%$

Adicional a los resultados obtenidos en la Escala Multifactorial de Bienestar Psicológico, la encuesta sociodemográfica evidenció que las personas solteras, en general, apreciaban la libertad que su situación implica, mientras que las personas casadas mencionaron el apoyo de sus parejas como un elemento fundamental en su satisfacción con su estado sentimental.

De igual manera, la satisfacción de las personas con respecto a su situación sentimental fue una variable que permitió entender las puntuaciones de los participantes; por ello, se realizó un análisis complementario que permitiera explicar las puntuaciones obtenidas. De acuerdo con esto, se observó una correlación baja entre el bienestar psicológico, junto con sus dimensiones, y la satisfacción con el estado sentimental actual, reportado en una escala de 1 a 10, siendo 1 bajo y 10 alto sin importar cuál fuera este (ver Tabla 6). 
Tabla 6

Correlación entre Bienestar Psicológico y Satisfacción con el estado Sentimental Actual

\begin{tabular}{|c|c|c|c|}
\hline & & Satisfacción & Tiempo de la relación \\
\hline \multirow{2}{*}{ Tiempo de la relación } & Coeficiente de correlación & -.032 & - \\
\hline & Sig. (bilateral) & .732 & - \\
\hline \multirow{2}{*}{ Autoaceptación } & Coeficiente de correlación & $.210^{\star \star}$ & .073 \\
\hline & Sig. (bilateral) & .002 & .436 \\
\hline \multirow{2}{*}{ Relaciones positivas } & Coeficiente de correlación & $.190^{\star \star}$ & .081 \\
\hline & Sig. (bilateral) & .006 & .385 \\
\hline \multirow{2}{*}{ Autonomía } & Coeficiente de correlación & $.225^{\star \star}$ & $.211^{*}$ \\
\hline & Sig. (bilateral) & .001 & .022 \\
\hline \multirow{2}{*}{ Dominio del entorno } & Coeficiente de correlación & $.274^{\star \star}$ & $.199^{*}$ \\
\hline & Sig. (bilateral) & .000 & .031 \\
\hline \multirow{2}{*}{ Crecimiento personal } & Coeficiente de correlación & $.180^{* \star}$ & -.012 \\
\hline & Sig. (bilateral) & .009 & .896 \\
\hline \multirow{2}{*}{ Propósito de vida } & Coeficiente de correlación & $.289^{\star \star}$ & .136 \\
\hline & Sig. (bilateral) & .000 & .143 \\
\hline \multirow{2}{*}{ Bienestar Psicológico } & Coeficiente de correlación & $.282^{\star \star}$ & .159 \\
\hline & Sig. (bilateral) & .000 & .086 \\
\hline
\end{tabular}

Es importante mencionar que no se encontraron tendencias correlativas entre el Bienestar Psicológico y variables como edad, sexo, tiempo de estado sentimental o tiempo de la relación para el caso de las personas casadas.

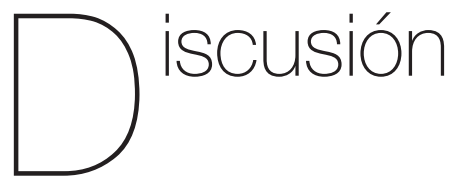

Los resultados evidenciaron que no existía diferencia significativa entre grupos con respecto al bienestar psicológico con una confianza de un 95\%, sin embargo, si se reducía la confianza, se determinaban diferencias entre personas solteras y personas que se mantenían en una relación; de acuerdo con esto, las personas solteras tenían puntuaciones levemente mayores en comparación con las personas casadas y las personas que se mantienen en una relación (ver Tabla 2). Estos datos son consistentes con información recolectada en la encuesta de datos sociodemográficos en donde las personas solteras mencionaban que uno de los aspectos que les agradaba de su situación sentimental era la libertad que la soltería implica.

No obstante, la mayoría de personas casadas en tal apartado mencionaron que el apoyo de su pareja era un aspecto fundamental en su relación, pese a ello, los resultados de la dimensión "relaciones positivas con otros" no se diferenciaron de las personas solteras y 
quienes mantenían una relación, lo cual no es consistente con los datos cualitativos recolectados, en donde se hipotetizaba que contar con una pareja estable y una convivencia permanente resultaría un elemento que aportaría al bienestar psicológico.

Teniendo en cuenta lo anterior, y al analizar cada dimensión de bienestar psicológico, es pertinente concluir que tanto el grupo de personas casadas, personas que se encuentran en una relación, como el grupo de personas solteras, se caracterizan por tener un nivel óptimo de autoaceptación, determinada por su madurez y su capacidad de autoactualizarse, así como en su propósito de vida, caracterizando a los tres grupos como direccionados e intencionados a una meta a futuro. Así mismo, las relaciones con otras personas en los tres grupos se pueden caracterizar como empáticas y de confianza.

No obstante, las personas casadas mantuvieron puntuaciones superiores a las personas solteras y aquellas que se encuentran en una relación, con respecto a dominio del entorno, lo cual los destaca como personas que tienen un sentido de dominio y competencia en la gestión del medio ambiente, que controlan un conjunto complejo de actividades externas y hacen uso eficaz de las oportunidades de su alrededor, siendo capaces de elegir o crear contextos adecuados (Ryff, 1989b), lo cual permite hipotetizar que las diferencias en estas puntuaciones se deban a la oportunidad de las personas casadas de desarrollar, en el matrimonio, ambientes propicios, tanto para el desarrollo personal como para la pareja, lo que no sucede en las personas que se mantienen en una relación, dada la ausencia de cohabitación.

De igual manera, la dimensión de crecimiento personal se diferenció en las personas que se encuentran en una relación en contraste con las personas casadas y solteras, caracterizándolas como personas que están en continuo desarrollo del propio potencial (Ryff, 1989b), lo que es consistente con el tiempo de relación de muchos participantes que, en general, era menor a 5 años, por lo cual puede evidenciarse un sentido de crecimiento tanto personal como de la pareja.

Por su parte, la dimensión de autonomía presentó diferencias significativas entre el grupo de personas casadas y solteras, en cuyo caso el segundo grupo tiene medias más altas, destacando su habilidad de autodeterminación, independencia y regulación conductual interna. Esto es consistente con la situación sentimental, pues las personas solteras no deben mediar con una pareja sobre varios elementos cotidianos y personales.

De acuerdo con las correlaciones encontradas, es importante mencionar que, aunque tienen magnitudes bajas, pueden evidenciar variables importantes con respecto al bienestar psicológico y el estado sentimental, como el tiempo de la relación, el cual se relacionó con la dimensión de autonomía y dominio del entorno de forma positiva. De la misma forma, la satisfacción con la situación sentimental se relacionó con todas las dimensiones de bienestar psicológico, por lo cual es una variable que puede verse relacionada con la forma en la que se percibe el bienestar en las relaciones sentimentales. 
De otro lado, de acuerdo con los antecedentes empíricos del modelo de Ryff, se conoce que el bienestar psicológico varía en función de la edad, el sexo y la cultura, mas no existía evidencia del modelo en función de la situación sentimental (Ryff, 1989b; Vázquez, \& Hervás, 2012), por lo cual, esta investigación evidenció que el bienestar psicológico puede ser una variable que varíe con respecto al estado sentimental. Puede concluirse que más allá del tipo de relación, la calidad del vínculo, la satisfacción con la relación y la forma como las personas perciben aspectos de su vida, como su situación sentimental, puede permear el bienestar psicológico, o bien sea, este puede permear la forma en la que las personas perciben sus propias relaciones.

De acuerdo con esto, se sugiere investigar a profundidad la percepción y evaluación de las personas sobre diferentes aspectos de su vida, incluyendo sus relaciones sociales o de pareja, lo que explicaría por qué las conclusiones de las investigaciones consultadas discrepaban para responder si casados o solteros presentan mayor o menor felicidad y bienestar.

Tales resultados dejan una serie de cuestionamientos que abren puertas a nuevas preguntas de investigación, por ejemplo: ¿El bienestar psicológico en función del matrimonio cambia a través de las culturas? ¿Los tipos de relaciones sociales aportan diferencialmente al bienestar psicológico? ¿Cuáles son las características de un buen matrimonio que logre aportar al bienestar psicológico? ¿Qué determina la calidad de un vínculo en una relación social?
Finalmente, se sugiere continuar en el estudio de tal constructo en relación con otro tipo de variables, teniendo en cuenta las limitaciones de la presente investigación, tales como tener una muestra significativamente mayor y que resulte representativa, así como lograr una equivalencia entre grupos, manteniendo control sobre variables como nivel de estudio, estrato socioeconómico y las categorías que puedan existir con respecto a los tipos de relaciones de pareja.

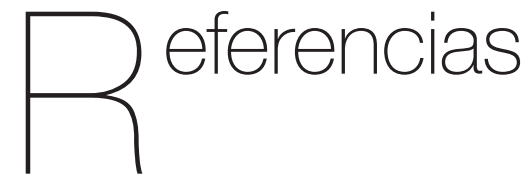

Azam, M. (2012). Determinants of psychological well-being and self-esteem in married and unmarried women. Pakistan Journal of Clinical Psychology, 11(2), 3-14.

Díaz, D., Rodríguez-Carvajal, R., Blanco, A., Moreno-Jiménez, B., Gallardo, I., Valle, C., \& Van Dierendonck, D. (2006). Adaptación española de las escalas de bienestar psicológico de Ryff. Psicothema, 18(3), 572-577.

Chapman, B., \& Guven, C. (2016). Revisiting the relationship between marriage and wellbeing: does marriage quality matter? Journal of Happiness Studies, 17(2), 533-551.

Erhabor, S., \& Neo J. (2013). How happy are married people? Psychological indicators of marital satisfaction of married men and women in gauteng province, South Africa. Gender \& behavior, 11(2), 5486-5498. 
García, I., \& Nader, F. (2009). Estereotipos masculinos en la relación de pareja. Enseñanza e Investigación en Psicología, 14(1), 37-45.

Girme, Y., Overall, N., Faingataa, S. \& Sibley, C. (2015). Happily Single: The Link Between Relationship Status and Well-Being Depends on Avoidance and Approach Social Goals. Social Psychological and Personality Science, 1(9), 122-130. doi: 10.1177/1948550615599828.

Jeong-Hwa, H. (2015). The problem group? Psychological wellbeing of unmarried people living alone in the Republic of Korea. Demographic Research, 32, 1299-1328.

Mikucka, M. (2016). The life satisfaction advantage of being married and gender specialization. Journal the marriage and family. Journal of marriage and family, 78(3), 759-779.

Musick, K. \& Bumpass, L. (2012). Reexamining the Case for Marriage: Union Formation and Changes in Well-Being. Journal of Marriage and Family, 74(1), 1-18.

Pozos, J., López, M., Rivera, S., Vargas, B., \& Reidl, L. (2013). Felicidad general y felicidad en la pareja: diferencias por sexo y estado civil. Enseñanza e Investigación en Psicología, 18(1), 69-84.

Ryff, C. (1989a). Beyond Ponce de Leon and Life Satisfaction: New Directions in Quest of Successful Ageing. International Journal of Behavioral Development, 12(1), 35-55.

Ryff, C. (1989b). Happiness is everything, or is it? Explorations on the meaning of psychological well-being. Journal of Personality and Social Psychology, 57, 1069-1081.
Ryff, C., Singer, B., \& Love, G. (2004). Positive health: Connecting well-being with biology. Philosophical Transactions of the Royal Society of London, 359, 1383-1394.

Shapiro, A \& Keyes, C. (2008). Marital Status and Social Well-Being: Are the Married Always Better Off? Social Indicators Research, 88, 329-346. DOI: 10.1007/ s11205-007- 9194-3.

Sheck, D. (1995). Marital quality and psychological well-being of married adults in a Chinese context. The Journal of Genetic Psychology, 156(1), 45-56.

Simó-Noguera, C., González-Sanjuán, M. E., Muñoz-Rodríguez, D., \& HernándezMonleón, A. (2015). El efecto del estado civil y de la convivencia en pareja en la salud. Reis. Revista Española de Investigaciones Sociológicas, 151, 141-165.

Soulsby, L. \& Bennett, K. (2015) Marriage and Psychological Wellbeing: The Role of Social Support. Psychology, 6, 1349-1359.

Superintendencia de Notariado y Registro. SNR. (2014). Boletín de estadísticas notariales, matrimonios civiles $y$ divorcios. Recuperado de https://www. supernotariado.gov.co/portalsnr/images/ archivosupernotariado/Estadisticas2014/ iii\%20boletin\%202014\%20notariado.pdf

Superintendencia de Notariado y Registro. SNR. (2017). En Colombia, por cada tres matrimonios hay un divorcio. Recuperado de https://www.supernotariado.gov.co/ PortalSNR/ShowProperty?nodeld=\%2FS NRContent\%2FWLSWCCPORTAL011496 11\%2F\%2FidcPrimaryFile\&revision=latestr eleased 
Szmulewicz, E. (2015). Terapia de pareja: un espacio intersubjetivo con arreglo triangular. Revista Argentina de Clínica Psicológica, 24(2), 101-110. Recuperado de http://www. redalyc.org/articulo.oa?id=281946783002

Tze-li, H. (2014). Theassociation between marital status and health: variation across age groups and dimensions of psychological well-being (Tesis de Doctorado). Florida State University, Florida, Estados unidos.
Vázquez, C., \& Hervás, G. (2012). Salud mental positiva: Del sintoma al bienestar. En C. Vázquez, \& G. Hervás, Psicología Positiva Aplicada (pp. 17-39). España: Desclee de Brower. 